\title{
Axial Diffusivity Is the Primary Correlate of Axonal Injury in the Experimental Autoimmune Encephalomyelitis Spinal Cord: A Quantitative Pixelwise Analysis
}

\author{
Matthew D. Budde, ${ }^{1}$ Mingqiang Xie, ${ }^{1}$ Anne H. Cross, ${ }^{2}$ and Sheng-Kwei Song ${ }^{1}$ \\ Departments of ${ }^{1}$ Radiology and ${ }^{2}$ Neurology, Washington University, St. Louis, Missouri 63110
}

The dissociation between magnetic resonance imaging (MRI) and permanent disability in multiple sclerosis (MS), termed the clinicoradiological paradox, can primarily be attributed to the lack of specificity of conventional, relaxivity-based MRI measurements in detecting axonal damage, the primary pathological correlate of long-term impairment in MS. Diffusion tensor imaging (DTI) has shown promise in specifically detecting axonal damage and demyelination in MS and its animal model, experimental autoimmune encephalomyelitis (EAE). To quantify the specificity of DTI in detecting axonal injury, in vivo DTI maps from the spinal cords of mice with EAE and quantitative histological maps were both registered to a common space. A pixelwise correlation analysis between DTI parameters, histological metrics, and EAE scores revealed a significant correlation between the water diffusion parallel to the white matter fibers, or axial diffusivity, and EAE score. Furthermore, axial diffusivity was the primary correlate of quantitative staining for neurofilaments (SMI31), markers of axonal integrity. Both axial diffusivity and neurofilament staining were decreased throughout the entire white matter, not solely within the demyelinated lesions seen in EAE. In contrast, although anisotropy was significantly correlated with EAE score, it was not correlated with axonal damage. The results demonstrate a strong, quantitative relationship between axial diffusivity and axonal damage and show that anisotropy is not specific for axonal damage after inflammatory demyelination.

\section{Introduction}

Magnetic resonance imaging (MRI) is essential in the diagnosis of multiple sclerosis (MS). Although the etiology of MS is still unknown, the pathological hallmark of MS, demyelinated lesions in the CNS white matter, can be visualized as focal signal intensity changes on T1- or T2-weighted MRI. Unfortunately, the size and number of lesions have had only modest correlations with neurological impairment in MS (Nijeholt et al., 1998; Barkhof, 1999). A number of pathological features typical of MS, including inflammation, demyelination, edema, and axonal damage, can all cause alterations of tissue relaxation properties (Markovic-Plese and McFarland, 2001). This has led to the clinicoradiological paradox in MS and has necessitated the development of a better biomarker of disease activity (Barkhof, 2002). There is strong evidence that axonal damage, not demyelination, is the major correlate of impairment in MS (Lovas et al., 2000; De Stefano et

Received Sept. 24, 2008; revised Dec. 30, 2008; accepted Jan. 23, 2009.

This work was supported by National Multiple Sclerosis Society Grants RG 3376-A-2/1 and CA 1012-A-13, National Institutes of Health (NIH) Grant R01-NS054194, and Washington University Small Animal Imaging Resource/ NIH Grant R24-CA83060. M.D.B. was supported by NIH-National Institute of Neurological Disorders and Stroke Predoctoral Fellowship F31-NS052057. A.H.C. was partially supported by the Manny and Rosalyn Rosenthal-Dr. John L. Trotter MS Center Chair in Neuroimmunology. We thank Bob Mikesell for immunization of the EAE mice, Dr. Joong Hee Kim for help with imaging, and Hsiao-Fang Liang for assistance with histological preparation.

Correspondence should be addressed to Dr. Sheng-Kwei Song, Biomedical MR Laboratory, Washington University School of Medicine, Campus Box 8227, 660 S. Euclid Avenue, St. Louis, M0 63110. E-mail: ssong@wustl.edu.

M. D. Budde's present address: Radiology and Imaging Sciences, National Institutes of Health Clinical Center, Bethesda, MD 20892.

DOI:10.1523/JNEUROSCI.4605-08.2009

Copyright $\odot 2009$ Society for Neuroscience $\quad$ 0270-6474/09/292805-09\$15.00/0 al., 2001; Petzold et al., 2005). Thus, a specific and sensitive noninvasive marker of axonal damage in MS would provide a significant advance relating radiological findings with neurological assessments.

Diffusion tensor imaging (DTI) is exceptionally sensitive to white matter pathology in MS and its animal model, experimental autoimmune encephalomyelitis (EAE). The directional diffusivities derived from DTI hold promise as specific markers of axonal damage and demyelination in MS and EAE. Radial diffusivity, the diffusion of water perpendicular to white matter fibers, increases in response to demyelination (Song et al., 2005) and dysmyelination (Song et al., 2002; Nair et al., 2005). In contrast, a decrease in axial diffusivity, the diffusion of water parallel to white matter fibers, is indicative of axonal damage (Song et al., 2003; Loy et al., 2007). It has been shown that axial diffusivity is highly correlated with axonal damage in EAE mice (DeBoy et al., 2007; Sun et al., 2007; Budde et al., 2008) and mouse models of other white matter injuries (Sun et al., 2006, 2008; Kim et al., 2007; Mac Donald et al., 2007). However, in a complex disease such as MS, which includes inflammation, demyelination, and axonal damage, it is unclear how accurately the directional diffusivities relate to specific pathologies. A robust, quantitative relationship between DTI parameters and the specific pathological features that they reflect has not been established. To overcome the clinicoradiological paradox in MS, the specificity of DTI in detecting white matter pathology is essential.

In this work, we present a quantitative pixel-by-pixel analysis between DTI parameters and histological markers of axonal 
damage, demyelination, and inflammation in the spinal cords of EAE mice. Both the DTI parameter maps and histological sections were registered to a common space using a novel registration and quantification technique. The results demonstrate that both DTI and histological markers of axonal damage were highly correlated with one another and that both measurements were highly correlated with hindlimb dysfunction. The results support axial diffusivity as a noninvasive biomarker of axonal damage in EAE and caution the use of anisotropy as the sole indicator of axonal damage.

\section{Materials and Methods}

Animals. All animal procedures were approved by the Washington University Animal Studies Committee. Thirty-two female mice underwent active immunization with $50 \mu \mathrm{g}$ of myelin oligodendrocyte glycoprotein peptide $\left(\mathrm{MOG}_{35-55}\right)$ emulsified in incomplete Freund's adjuvant with 50 $\mu \mathrm{g}$ of Mycobacterium tuberculosis for EAE induction. A cohort of eight female mice served as controls (Table 1 ). Mice were evaluated daily using a published $0-5$ scoring system: 0 , normal; 1 , limp tail; 2 , hindlimb weakness sufficient to impair righting; 3 , one limb paralyzed; 4 , two limbs paralyzed; 5, more than two limbs paralyzed or the animal is moribund (Cross et al., 1994). All mice underwent imaging at $>25 \mathrm{~d}$ after immunization (Fig. 1).

Magnetic resonance imaging. Mice were anesthetized using 5\% isoflurane in oxygen, fitted with a custom nose cone to deliver $1 \%$ isoflurane in oxygen for maintenance, and placed in a custom holder designed to isolate spinal cord motion (Kim et al., 2006). Core temperature was maintained at $37^{\circ} \mathrm{C}$ with circulating warm water. A $9 \mathrm{~cm}$ inner diameter Helmholtz coil and a $9 \times 16 \mathrm{~mm}$ saddle surface coil were used as the radio frequency transmitter and receiver, respectively. Both coils used diodebased active decoupling (Mispelter et al., 2006). The entire preparation was placed in a $4.7 \mathrm{~T}$ Oxford Instruments magnet equipped with a $10 \mathrm{~cm}$ inner diameter, actively shielded Magnex gradient coil $(60 \mathrm{G} / \mathrm{cm}, 200 \mu \mathrm{s}$ rise time). The magnet, gradient coil, and Techron gradient power supply were interfaced with a Varian UNITY-INOVA console controlled by a Sun Microsystems Blade 1500 workstation. The ilium was identified on coronal scout images and was used as an internal reference to identify the vertebral segments. Six transverse slices were collected covering segments T13-L2 using a Stejskal-Tanner spin-echo diffusion-weighted sequence (Stejskal and Tanner, 1965), with the following acquisition parameters: repetition time, $\geq 1500 \mathrm{~ms}$ (determined by respiratory rate); echo time, $43 \mathrm{~ms}$; number of excitations, 4; slice thickness, $1.0 \mathrm{~mm}$; field of view, 1 $\mathrm{cm}^{2}$; data matrix, $128 \times 128$ (zero filled to $256 \times 256$ ). Diffusionweighted images were collected using respiratory gating, and diffusion sensitizing gradients were applied in six orientations: $(G x, G y, G z)$ of $(1,1,0),(1,0,1),(0,1,1),(-1,1,0),(0,-1,1)$, and $(1,0,-1)$ with a gradient strength of $11.25 \mathrm{G} / \mathrm{cm}$, duration $(\delta)$ of $10 \mathrm{~ms}$, and separation $(\Delta)$ of 25 $\mathrm{ms}$, to obtain a $b$ value of $785 \mathrm{~s} / \mathrm{mm}^{2}$. Acquisition time was $\sim 2 \mathrm{~h}$. DTI parameter maps were calculated for $\lambda_{\|}, \lambda_{\perp}$, and relative anisotropy (RA) as described previously (Song et al., 2002).

Histological analysis. Immediately after imaging, mice were perfusion fixed with $0.01 \mathrm{~m}$ PBS, followed by $4 \%$ paraformaldehyde in PBS. Vertebral columns were excised, fixed overnight, and decalcified for $48 \mathrm{~h}$. Fixed spinal cords were embedded in paraffin and cut on a sliding microtome at a thickness of $3 \mu \mathrm{m}$. The ilium used as a reference in the MR images was similarly used to ensure the histological sections were obtained from the DTI slice of interest. Sections were deparaffinized and rehydrated, and antigen retrieval was performed with sections in $1 \mathrm{~mm}$ EDTA at $95-100^{\circ} \mathrm{C}$ water bath. Sections were blocked in $2 \%$ blocking buffer (Invitrogen) for $1 \mathrm{~h}$ at room temperature. Adjacent sections were incubated with either polyclonal anti-myelin basic protein (anti-MBP; 1:1000; Sigma) or monoclonal anti-phosphorylated neurofilament $\mathrm{H}$ (SMI31; 1:5000; Sternberger Monoclonals) at $4^{\circ} \mathrm{C}$ overnight. After rinse, goat anti-mouse or rabbit IgG conjugated with cyanine 3 (1:300; Jackson ImmunoResearch) were applied to visualize immunoreactive materials. After washing, sections were covered in Vectashield Mounting Medium with 4',6'-diamidino-2-phenylindole (DAPI) (Vector Laboratories). Histological sections were prepared from 27 EAE and three control mice
Table 1. Summary of mice used in the analysis

\begin{tabular}{llll}
\hline Group & EAE score & No. of mice for DTI & No. of mice for histology \\
\hline Control & 0 & 8 & 3 \\
EAE & 0 & 9 & 7 \\
& 1 & 5 & 4 \\
& 2 & 9 & 8 \\
& 3 & 3 & 2 \\
& 4 & 6 & 6 \\
\hline
\end{tabular}

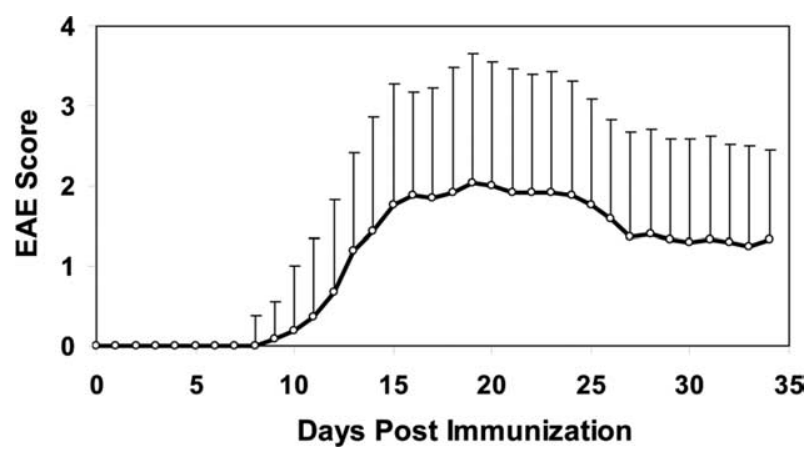

Figure 1. Clinical course of EAE mice. Mean daily EAE score for 32 mice. Error bars represent SD. All mice underwent DTI in the chronic phase ( $>25 \mathrm{~d}$ after immunization).

(Table 1). Digital histological images were acquired at $4 \times$ magnification on a Nikon 80i fluorescence microscope. Sections stained for the same primary antibody were captured with identical excitation intensity and exposure time settings.

Image registration. Registration of the spinal cord $\mathrm{MR}$ images was performed using a manual, two-dimensional registration procedure with two iterations. Maps of radial diffusivity provided the best contrast to differentiate white matter from gray matter and CSF. Therefore, radial diffusivity maps were used to register the datasets. Each slice was independently rotated and translated to center the spinal cord within the image field of view. To generate a template, the radial diffusivity maps were averaged across all mice for each slice position. To account for differences in the size of the spinal cord between animals, radial diffusivity maps were spatially scaled to minimize the difference between each image and the template average. The other DTI parameter maps were subsequently transformed with the derived registration parameters (Fig. 2).

The registration of histological images to MR images was performed using a modification of the previously described method (Budde et al., 2007). Sixteen corresponding landmarks were manually placed on the average radial diffusivity map and each of the digital histological images (Fig. $3 A, B$ ). Landmarks included the anterior and posterior arteries, prominent white and gray matter interfaces, and points along the perimeter of the cord. A thin-plate spline warp (Bookstein, 1989) deformation grid was computed and superimposed on the histological image in which each of the grid regions represented the size of a single pixel on the MR image. Quantitative histological maps were derived by computing the area fraction of positive staining, an approach commonly used to quantify immunohistochemistry sections (Matos et al., 2006; Kokolakis et al., 2008). Briefly, each digital histological image was first thresholded with a value derived from the pixel intensities of the surrounding spinal nerves. The spinal nerves are not affected in MOG-induced EAE, and this ensured an objective, internal reference for each section. The thresholds were empirically chosen for each staining type as the value that resulted in a mean area threshold of 50\% across all pixels from all animals, which minimized floor and ceiling effects. The threshold for SMI31-stained sections was equal to the mean +0.25 SD intensity of the spinal nerves, whereas the threshold for MBP and DAPI stains were set as the mean + $4 \mathrm{SD}$ and the mean $+1 \mathrm{SD}$, respectively. Each pixel in the final, downsampled image represented the area fraction of positive staining within each grid square (Fig. $3 E$ ). All image processing procedures were written 

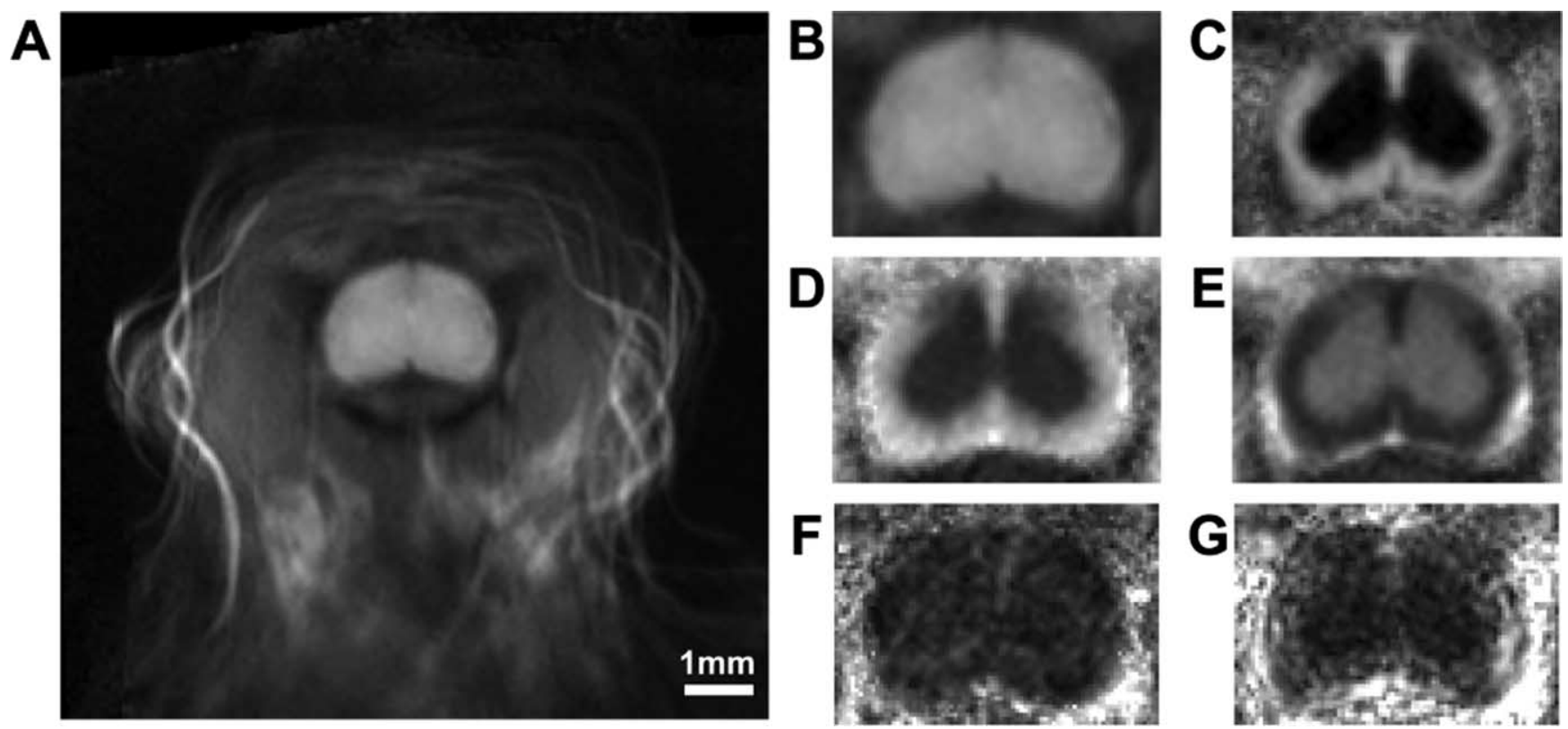

Figure 2. Registration of DTI parameter maps. DTI parameter maps were manually registered to a common space using rotations, translations, and global scaling. The average T2-weighted ( $b=$ 0) image from eight control mice $(\boldsymbol{A})$ shows that the spinal cord is well aligned after registration. The average T2-weighted $(\boldsymbol{B})$, RA $(\boldsymbol{C})$, axial $(\boldsymbol{D})$, and radial $(\boldsymbol{E})$ parameter maps demonstrate the preservation of white matter borders after the registration. The maps of the coefficient of variation for axial $(\boldsymbol{F})$ and radial $(\boldsymbol{G})$ diffusivity further demonstrate the reproducibility of the in vivo DTI data and the registration accuracy.
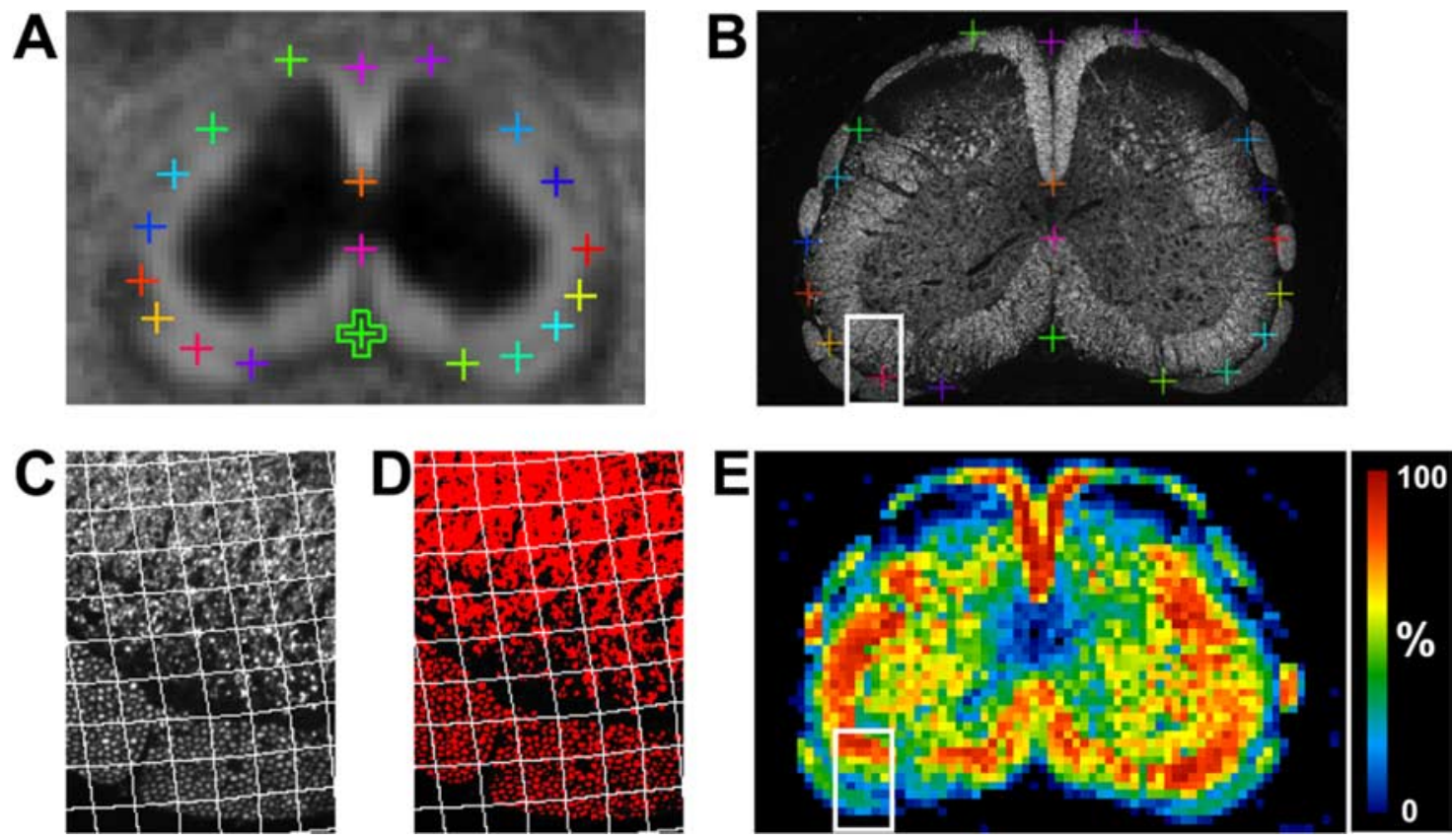

Figure 3. Registration and quantification of histological sections. Corresponding landmarks were placed on the average RA map $(\boldsymbol{A})$ and each of the digitized histological images ( $\boldsymbol{B} ;$; $\mathrm{SMI} 31$ shown here). Landmarks were manually placed along the white matter border and along prominent white and gray matter interfaces. A thin-plate spline deformation grid was derived and overlaid on the histological image ( $\boldsymbol{C}$; magnified view) in which each square of the grid corresponds to a single pixel on the MRI image. The histological image was segmented (D) into foreground (red) and background (black) using a threshold value derived from the pixel intensities of the surrounding spinal nerves, which are not affected in MOG-induced EAE. The area fraction of foreground staining for each square of the grid was computed to produce a quantitative histological image $(\boldsymbol{E})$ that was registered to the MR images.

in Java and implemented as plugins in NIH ImageJ. A modified version of the UnwarpJ plugin (Sorzano et al., 2005) facilitated the placement of image landmarks. Regions of interest encompassing the entire white matter were manually traced on the average radial diffusivity map and applied to other maps.

Statistical analysis. Both a region of interest and a pixel-by-pixel analysis were performed. Spearman's rank correlation coefficients were used to correlate EAE scores with either DTI or histological measures. Correlations between DTI parameters and histological measures used a Pearson's correlation. A multivariate linear regression was used to investigate the effects of confounding variables on the correlation between axial diffusivity and SMI31. In the pixel-bypixel analysis, statistical tests were performed for each pixel, and the resulting $p$ values were corrected for multiple comparisons by controlling for the false discovery rate (Benjamini and Hochberg, 1995). Statistical maps were thresholded at a corrected $p$ value of 0.05 . All statistical procedures were performed using the Statistics Toolbox of Matlab (MathWorks). 


\section{Results}

Thirty-two mice with a range of EAE scores (Table. 1) underwent in vivo DTI in the chronic phase of EAE, 1 month after immunization (Fig. 1). Eight mice served as controls and underwent DTI at age-matched time points. The average DTI parameter maps from control mice show clear tissue boundaries following manual registration to one another (Fig. 2 ). The maps of the coefficient of variation for axial and radial diffusivity (Fig. $2 F, G)$ reveal no distinct boundaries between white and gray matter, which demonstrated the suitability of the registration procedure for assessing all mice. The registered DTI parameter maps from all mice were grouped according to their EAE score on the day of imaging and averaged (Fig. 4). A decrease in axial diffusivity in the ventrolateral white matter was related to the severity of EAE. The pixelwise correlation analysis between EAE scores and axial diffusivity revealed a significant negative correlation throughout nearly the entire white matter and the most posterior region of the dorsal white matter (Fig. 5). The pattern was consistent throughout the four image slices from the lumbar spinal cord. In contrast, radial diffusivity of white matter was increased in mice with EAE compared with control mice, but no consistent pattern was observed relating to EAE score (Fig. 4). Only a few pixels along the periphery of the white matter were significantly correlated with impairment in EAE (Fig. 5). RA also decreased with increasing impairment (Figs. 4, 5).

In addition to registering MR images to one another, histological sections from 30 mice were registered to the DTI space using a novel registration and quantification scheme (Fig. 3). The area fraction of positive staining was used to quantify the downsampled histological sections. Immunohistochemistry staining for phosphorylated neurofilaments (SMI31) is indicative of intact axons, and a decrease in SMI31 staining was related to the degree of impairment (Fig. 6). The pattern is much more heterogeneous than that seen with DTI and reflects both intra-animal variation as well as variations inherent in immunohistological preparation. SMI31 staining was significantly correlated with EAE score in a large portion of the ventrolateral white matter (Fig. 7). MBP staining for the presence of myelin revealed differences between mice with EAE and control mice (Fig. 6), and MBP staining correlated with clinical score only along the white matter periphery (Fig. 7). DAPI staining showed an increase in cellular infiltration in mice with severe EAE, particularly along the most dorsal white matter (Fig. 6). A few

EAE CS4 spinal segment $L 2$.
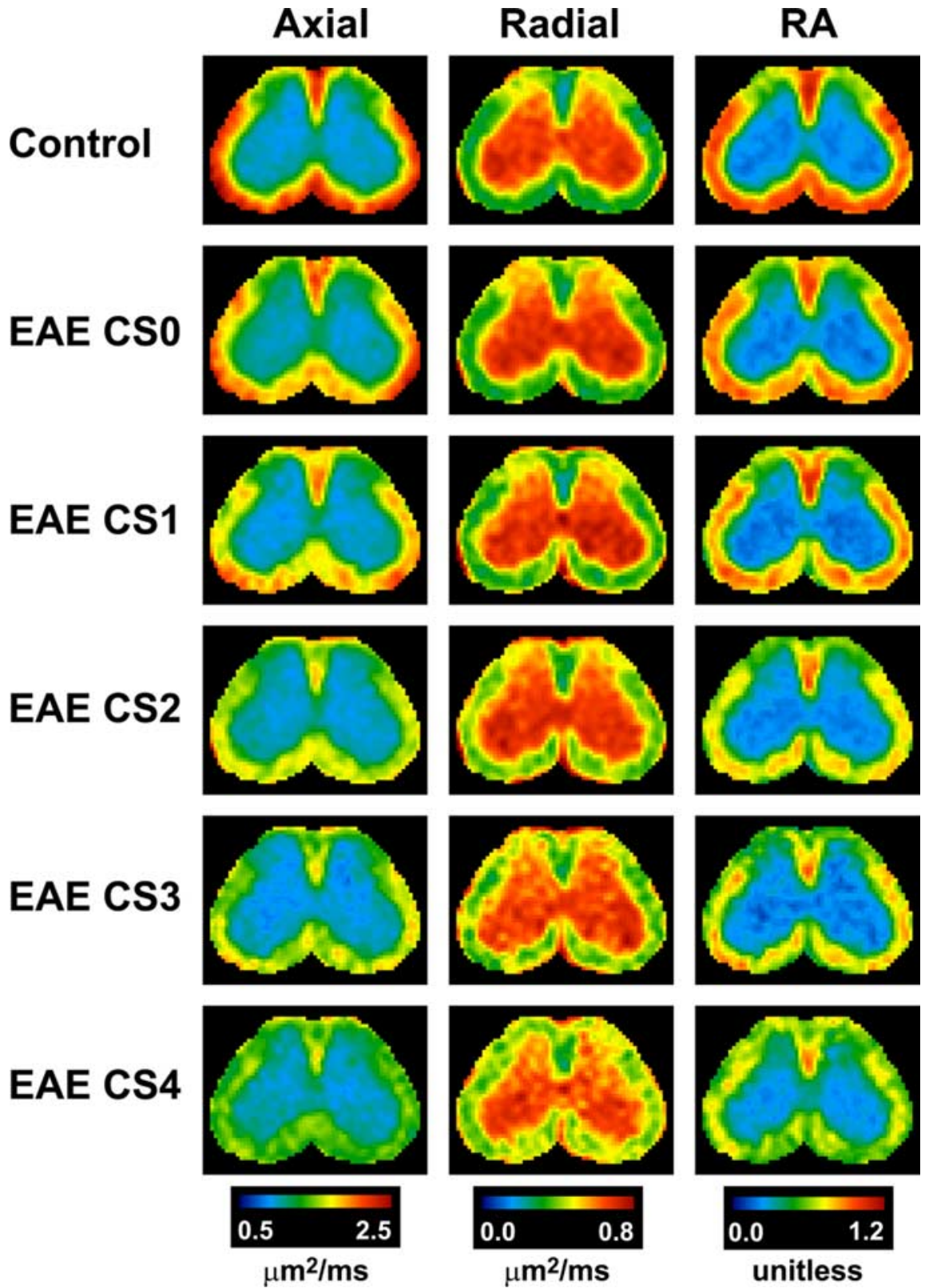

Figure 4. Mean DTI parameter maps grouped by EAE clinical score. The spinal cord white matter of mice with increasing severity of EAE (from top to bottom) shows a decrease in axial diffusivity that scales with the degree of impairment. The decrease was prominent in nearly all of the ventrolateral white matter as well as in the most posterior portion of the dorsal white matter. Radial diffusivity was increased in the ventrolateral white matter in mice with EAE, but there was no consistent pattern associated with the severity of EAE. RA decreased with increasing severity, reflecting its dependence on axial diffusivity. Images are from

sparse pixels correlated with EAE score (Fig. 7), and these appeared to correspond to regions of MBP decreases. The inflammatory cells were primarily composed of lymphocytes and monocytes/macrophages based on the morphological assessment of corresponding hematoxylin and eosin sections (data not shown).

Pixel-by-pixel correlations between the different histological markers revealed a strong correlation between inflammation, demyelination, and axonal damage in the spinal cord white matter (Fig. $8 \mathrm{~A}$ ). Correlations between the DTI parameters and histological measures revealed that axial diffusivity was significantly correlated with only axonal damage (Fig. $8 B$ ). Importantly, axial diffusivity was not significantly corre- 


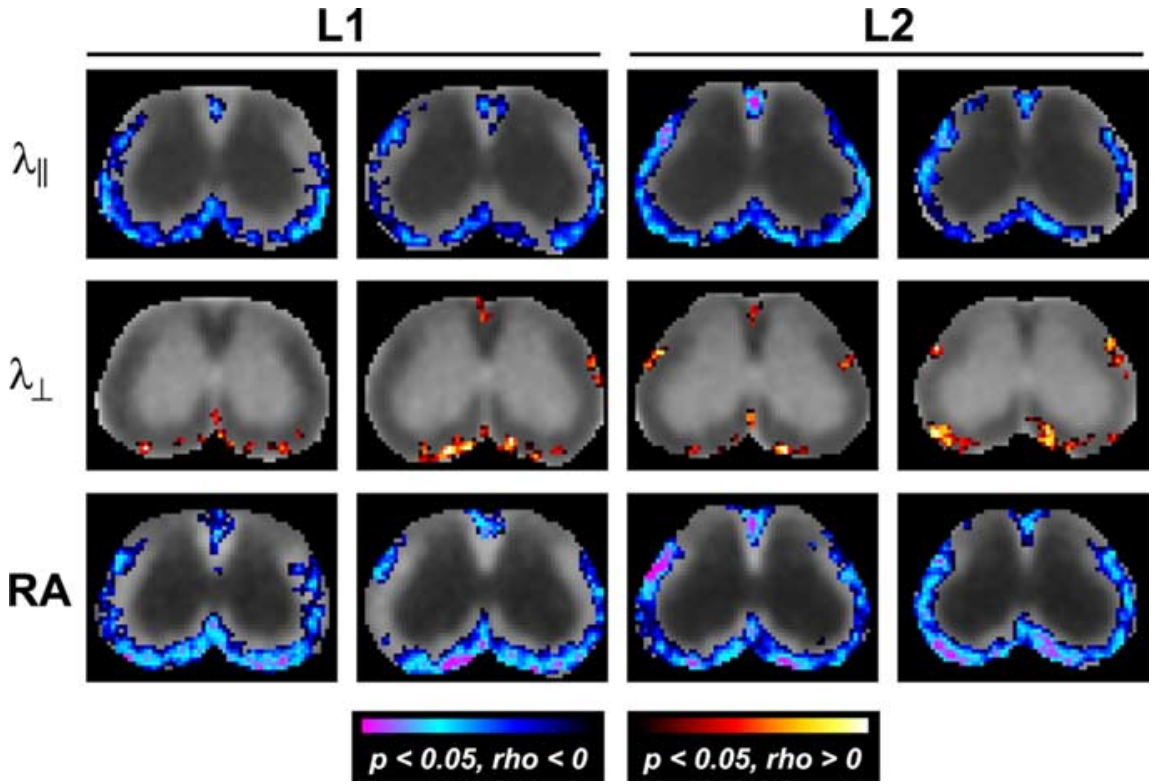

Figure 5. Pixelwise correlations between DTI parameters and EAE scores. Four adjacent slices of lumbar spinal cord segments $\mathrm{L} 1$ and $\mathrm{L} 2$ are shown. Axial diffusivity and RA were significantly correlated with EAE score in nearly the entire ventrolateral white matter and the most dorsal portion of the dorsal white matter. Radial diffusivity was significantly correlated with EAE score in only a few regions along the periphery of the ventrolateral white matter. Correlation (Spearman's $r$ ) maps were thresholded at $p<$ 0.05 and corrected for multiple comparisons, and significant voxels were overlaid on the average DTI parameter maps from all mice.

Table 2. Mean correlation coefficients from ventrolateral white matter region of interest analysis

\begin{tabular}{|c|c|c|c|c|c|}
\hline & EAE score* & SMI31 & MBP & DAPI & $\lambda_{\|}$ \\
\hline SMI31 & $-0.42(0.009)$ & & & & \\
\hline MBP & $-0.41(0.011)$ & $0.27(0.069)$ & & & \\
\hline DAPI & $0.52(0.003)$ & $-0.41(0.010)$ & $-0.73(<0.001)$ & & \\
\hline$\lambda_{\|}$ & $-0.73(<0.001)$ & $0.56(<0.001)$ & $0.25(0.08)$ & $-0.48(0.003)$ & \\
\hline$\lambda_{\perp}^{\prime \prime}$ & $0.67(<0.001)$ & $-0.22(0.21)$ & $-0.16(0.20)$ & $0.07(0.34)$ & $-0.49(0.006)$ \\
\hline $\mathrm{RA}$ & $-0.76(<0.001)$ & $0.48(0.003)$ & $0.22(0.12)$ & $-0.31(0.046)$ & \\
\hline
\end{tabular}

Pearson's $r$ correlations ( $p$ value), except when indicated (* indicates Spearman's rho correlation).

Table 3. Multivariate linear regression region of interest analysis of predictors of axial diffusivity

\begin{tabular}{llll}
\hline & SMI31 & MBP & DAPI \\
\hline$\lambda_{\|}$ & $0.45(0.007)$ & $-0.16(0.20)$ & $-0.35(0.03)$ \\
\hline
\end{tabular}

Partial $r$ values ( $p$ values).

lated with cellular infiltration. Furthermore, RA was not correlated with SMI31 content. The other pairwise combinations of DTI parameters (axial, radial, or RA) with histological measures (SMI31, MBP, or DAPI) had little or no correlation. The multivariate linear regression analysis (Fig. $8 C$ ) revealed that the correlation between axial diffusivity and SMI31 was partially maintained when MBP and DAPI were included as covariates in the regression model.

The region of interest analysis revealed significant correlations among most of the measures (Table 2). All of the histological and DTI measures were correlated with EAE score. Inflammation was correlated with both axonal damage and demyelination. As in the pixelwise analysis, SMI31 staining had the highest correlation with axial diffusivity. This correlation maintained its significance when MBP and DAPI were included as regressors in a multivariate analysis. (Table 3).

\section{Discussion}

The contribution of different pathological features of white matter injury to the DTI changes seen in MS and EAE have not been fully resolved. In this work, the registration of DTI and quantitative histological maps from multiple animals to a common space permitted a pixelwise analysis of the intricate relationship between DTI, pathology, and impairment in EAE. Axial diffusivity was highly correlated with both EAE scores and histological measures of axonal damage. Axonal damage has been shown to be a major correlate of neurological disability in MS (De Stefano et al., 2001) and EAE (Wujek et al., 2002; Papadopoulos et al., 2006), and the specificity of axial diffusivity for axonal damage is a significant advance in better assessing white matter pathology noninvasively. Importantly, the presence of cellular infiltrates, or inflammation, did not significantly correlate with axial diffusivity, as this has been a concern with DTI (Schmierer et al., 2007). However, the inclusion of MBP and DAPI as covariates in the model decreased the correlation between SMI31 and axial diffusivity. Thus, inflammation could decrease the specificity to axonal damage after inflammatory demyelination. It should be pointed out that the current work was performed in mice with chronic EAE. The intense inflammation occurring during the onset phase of EAE could have a greater influence on the diffusivity measures than in chronic EAE. Previous reports have described a relationship between axial diffusivity and axonal damage after demyelination in the rodent spinal cord using either a region of interest analysis approach (Budde et al., 2008) or the parcellation of white matter tracts using diffusion tractography (DeBoy et al., 2007). However, EAE has substantial variability in both the degree of impairment that different animals experience and the distribution of lesions within the spinal cord. The present work sought to resolve the connection between disability and lesion location using a pixel-by-pixel analysis. This approach revealed a high degree of correspondence between histological measures and DTI measures of axonal damage. Both histology and DTI demonstrated that axonal damage is widespread in EAE and hindlimb dysfunction does not relate to localized damage to specific tracts within the spinal cord.

DTI-measured axonal damage was evident in a large portion of the EAE spinal cord white matter, not just within the discrete demyelinating lesions that are the hallmark of MS and EAE. In the murine cord, axons responsible for gross motor movements are distributed throughout the ventral and lateral white matter (Loy et al., 2002), and the extensive correlations with EAE score highlight the correspondence between impairment and axonal damage occurring in white matter tracts known to relate to specific neurological functions. Axonal damage in the normal-appearing 
white matter (NAWM) in MS can be a significant pathological feature (Lovas et al., 2000), and many MRI abnormalities have been seen in the NAWM that appear to better relate to impairment (Charil et al., 2003; Miller et al., 2003). Significant axonal damage is seen in the spinal cords in MS (Bjartmar et al., 2000; Sathornsumetee et al., 2000), and damage to the spinal cord white matter is likely to lead to detectable motor deficits.

The relationship between demyelination and radial diffusivity is somewhat more complicated for a variety of reasons. For instance, it has not been well defined whether radial diffusivity is specific to demyelination in the presence of significant axonal damage (Sun et al., 2006) or inflammation. Although the connection between radial diffusivity and demyelination has been shown in several demyelinating and dysmyelination models, radial diffusivity may not be as specific in the presence of other pathological changes. In addition, MBP is present within both intact myelin and myelin debris, and staining for MBP does not clearly delineate intact from injured myelin. Whereas axons span the entire long lengths of the cord, demyelination is a focal, with lesions appearing as discrete regions within white matter. The thickness of a DTI slice may lead to partial volume effects that reduce the sensitivity of DTI to demyelination. Furthermore, the selection of the diffusion weighting in the present work may be less sensitive to myelin injury than to axonal injury. Increasing diffusion times or diffusion weighting may increase the sensitivity to demyelination (Biton et al., 2005; Nair et al., 2005).

Anisotropy, a summary parameter derived from DTI, was also highly correlated with EAE score. More importantly, it was not significantly correlated with histological markers of axonal damage or demyelination. Anisotropy is often used as a marker of white matter injury in MS and other white matter diseases and injuries. However, the interpretation of a decrease in anisotropy is not straightforward. Because anisotropy is derived from the directional diffusivities (eigenvalues) of the diffusion tensor, anisotropy will decrease after either a decrease in axial diffusivity or an increase in radial diffusivity. Thus, anisotropy will decrease after axonal damage, demyelination, or both. As shown here, anisotropy is not specific for axonal damage alone. Therefore, caution should be used when interpreting a de-

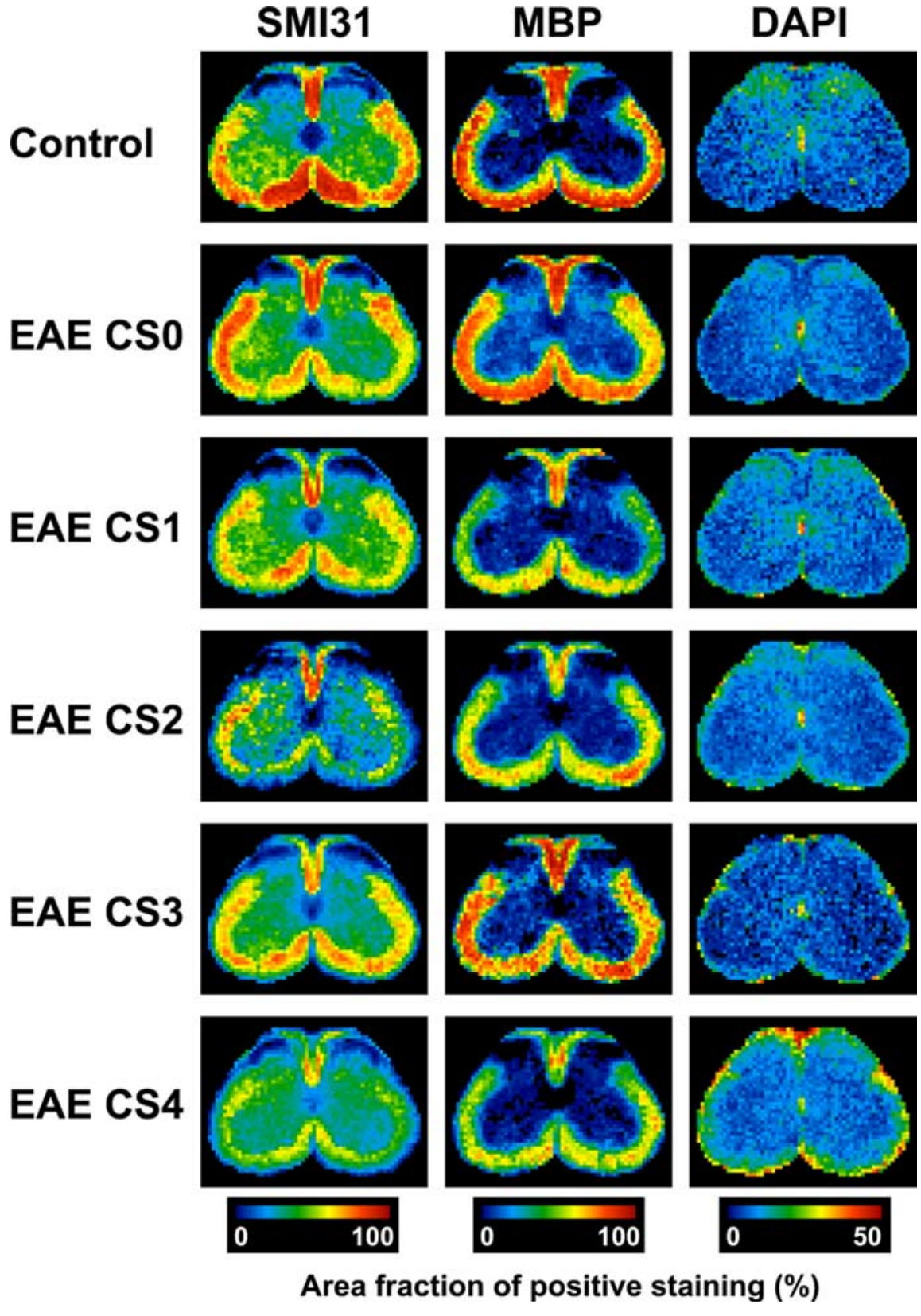

Figure 6. Mean quantitative histological maps grouped by EAE score. SMI31 staining (left) reveals a loss of intact axons that scales with the severity of EAE (from top to bottom). MBP staining for intact myelin is decreased in the EAE spinal cord white matter, but no consistent relationship with EAE score is evident. DAPI staining is indicative of cellularity and is particularly increased in certain regions in mice with clinical scores of 4 (bottom).

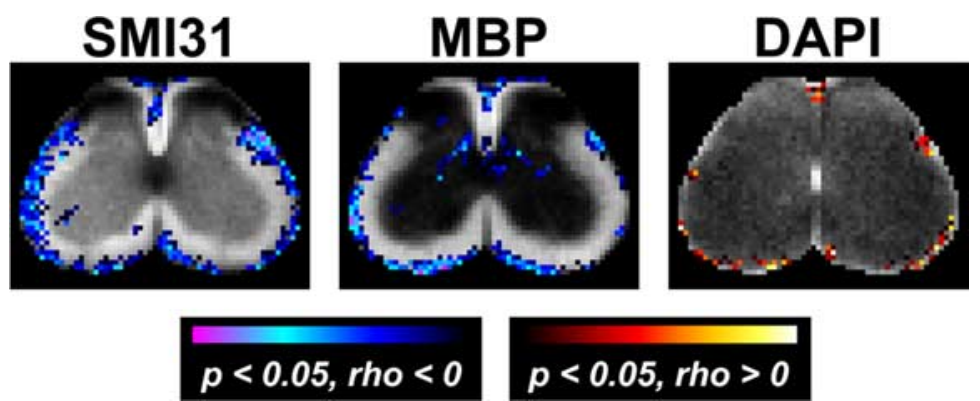

Figure 7. Pixelwise correlations between quantitative histological measures and EAE score. SMI31 staining was significantly correlated with EAE score in the majority of the spinal cord white matter. MBP staining was significantly correlated with EAE score along the white matter periphery. DAPI staining demonstrated only few pixels reaching significance. Correlation (Spearman's $r$ ) maps were thresholded at $p<0.05$, corrected for multiple comparisons, and overlaid on the mean registered histological maps from all mice. 

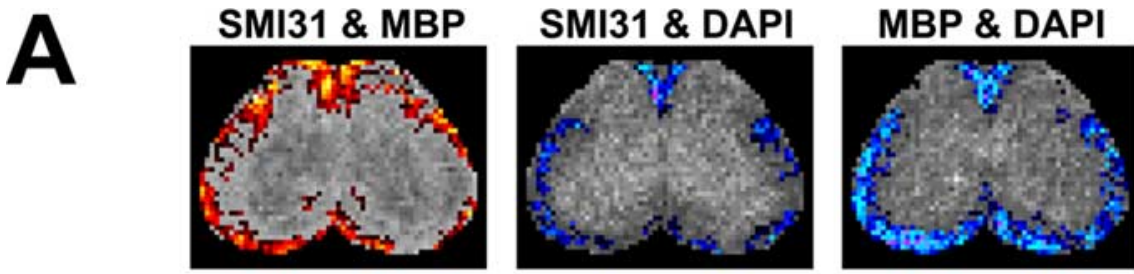

B
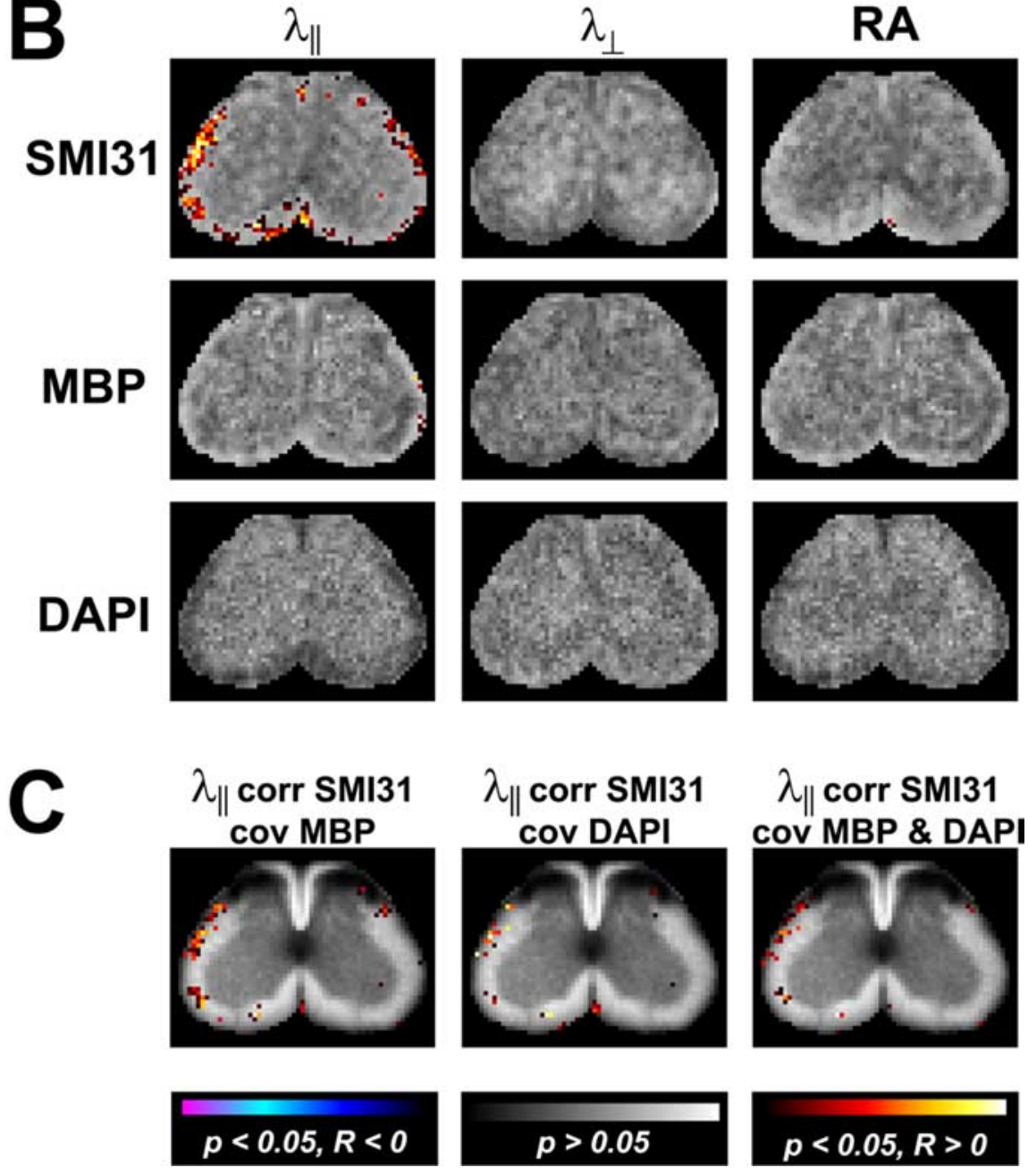

Figure 8. Pixelwise correlations between histological and DTI measures. SMI31, MBP, and DAPI were highly correlated with one another $(\boldsymbol{A})$, reflecting the intimate relationship between inflammation, demyelination, and axonal damage in EAE. Despite the interrelation, only axial diffusivity was highly correlated with SMI31 staining $(\boldsymbol{B})$. To address the potential confounds affecting the correlation between axial diffusivity and SMI31, MBP and DAPI were included as covariates in the regression model (C). Axial diffusivity was still significantly correlated with axonal damage, although less 50 . In all panels, significant pixels were thresholded at $p<0.05$ (corrected for multiple comparisons) and are shown in color overlaid on the correlation (Pearson's $r$ ) maps, except for C, which is overlaid on the average SMI31 map. The figure legend applies to all panels.

crease in anisotropy. Additionally, in regions of low anisotropy such as the gray matter, which can also contain lesions in MS (Vrenken et al., 2006), the directional diffusivities may not be adequately resolved. Importantly, the directional diffusivities were proposed specifically for interpreting white matter injury, and mean diffusivity is more appropriate in gray matter.

It is important to note that the specificity of DTI shown here was performed in the spinal cord, in which the white matter fibers have a uniform orientation. Although the human brain has regions of white matter that are highly ordered, such as the corpus callosum, the effect of multiple directions of fibers within a single DTI voxel could complicate the interpretation of diffusivity changes. Furthermore, the timing of the injury could be quite important in relating DTI changes with pathology. Specifically, axial diffusivity was found to decrease acutely after optic neuritis (Naismith et al., 2008). However, it had increased to well above control levels 1 year later. Longterm studies in animals may be needed to fully appreciate the time-dependant changes in histopathology that exist in the human situation. Nonetheless, changes in the directional diffusivities have been reported in many white matter disorders and have even been shown to relate to cognitive abilities (Dougherty et al., 2007; Qiu et al., 2008). Thus, it is believed that the specificity of axonal damage shown here may help resolve the interpretation of DTI changes in the human brain and spinal cord.

Although registering the spinal cord using manual procedures is time consuming (Kornelsen and Stroman, 2004), intensity- and surface-based approaches have not been optimized for application to the spinal cord despite their prominent use for the brain. As MRI of the spinal cord becomes more prevalent and resolution is improved (Hesseltine et al., 2006; Jeong et al., 2006; Kharbanda et al., 2006), registration methods will be needed and will presumably become more common. Other methods have been shown to register histological sections and MR images (Jacobs et al., 1999; Meyer et al., 2006). However, it is essential to use unbiased registration approaches when validating the connection between two modalities. With this goal in mind, landmark- and surfacebased approaches are more appropriate compared with intensity-based registration approaches. The current work presents a novel method to register and quantify histological sections that permits a direct, pixelwise comparison between histology and MR images. We believe comparisons of this nature are essential to establish a direct relationship between imaging modalities and the gold standard histological markers of pathology.

\section{Conclusions}

A decrease in axial diffusivity derived from DTI has been shown to be a specific marker of axonal damage in the spinal cord white matter of mice with EAE. DTI-detected axonal damage occurred in nearly all of the ventrolateral white matter and was not confined to the discrete demyelinating lesions. Thus, detection of axonal damage using DTI in both lesions and NAWM provides 
the necessary sensitivity and specificity of a noninvasive biomarker that may ultimately prove useful in overcoming the clinicoradiological paradox in MS.

\section{References}

Barkhof F (1999) MRI in multiple sclerosis: correlation with expanded disability status scale (EDSS). Mult Scler 5:283-286.

Barkhof F (2002) The clinico-radiological paradox in multiple sclerosis revisited. Curr Opin Neurol 15:239-245.

Benjamini Y, Hochberg Y (1995) Controlling the false discovery rate: a practical and powerful approach to multiple testing. J R Stat Soc B Methods 57:289-300.

Biton IE, Mayk A, Kidron D, Assaf Y, Cohen Y (2005) Improved detectability of experimental allergic encephalomyelitis in excised swine spinal cords by high b-value q-space DWI. Exp Neurol 195:437-446.

Bjartmar C, Kidd G, Mörk S, Rudick R, Trapp BD (2000) Neurological disability correlates with spinal cord axonal loss and reduced $\mathrm{N}$-acetyl aspartate in chronic multiple sclerosis patients. Ann Neurol 48:893-901.

Bookstein FL (1989) Principal warps: thin-plate splines and the decomposition of deformations. IEEE Trans Pattern Anal Mach Intell 11:567-585.

Budde MD, Kim JH, Liang HF, Schmidt RE, Russell JH, Cross AH, Song SK (2007) Toward accurate diagnosis of white matter pathology using diffusion tensor imaging. Magn Reson Med 57:688-695.

Budde MD, Kim JH, Liang HF, Russell JH, Cross AH, Song SK (2008) Axonal injury detected by in vivo diffusion tensor imaging correlates with neurological disability in a mouse model of multiple sclerosis. NMR Biomed 21:589-597.

Charil A, Zijdenbos AP, Taylor J, Boelman C, Worsley KJ, Evans AC, Dagher A (2003) Statistical mapping analysis of lesion location and neurological disability in multiple sclerosis: application to 452 patient data sets. Neuroimage 19:532-544.

Cross AH, Misko TP, Lin RF, Hickey WF, Trotter JL, Tilton RG (1994) Aminoguanidine, an inhibitor of inducible nitric oxide synthase, ameliorates experimental autoimmune encephalomyelitis in SJL mice. J Clin Invest 93:2684-2690.

DeBoy CA, Zhang J, Dike S, Shats I, Jones M, Reich DS, Mori S, Nguyen T, Rothstein B, Miller RH, Griffin JT, Kerr DA, Calabresi PA (2007) High resolution diffusion tensor imaging of axonal damage in focal inflammatory and demyelinating lesions in rat spinal cord. Brain 130:2199-2210.

De Stefano N, Narayanan S, Francis GS, Arnaoutelis R, Tartaglia MC, Antel JP, Matthews PM, Arnold DL (2001) Evidence of axonal damage in the early stages of multiple sclerosis and its relevance to disability. Arch Neurol 58:65-70.

Dougherty RF, Ben-Shachar M, Deutsch GK, Hernandez A, Fox GR, Wandell BA (2007) Temporal-callosal pathway diffusivity predicts phonological skills in children. Proc Natl Acad Sci U S A 104:8556-8561.

Hesseltine SM, Law M, Babb J, Rad M, Lopez S, Ge Y, Johnson G, Grossman RI (2006) Diffusion tensor imaging in multiple sclerosis: assessment of regional differences in the axial plane within normal-appearing cervical spinal cord. Am J Neuroradiol 27:1189-1193.

Jacobs MA, Windham JP, Soltanian-Zadeh H, Peck DJ, Knight RA (1999) Registration and warping of magnetic resonance images to histological sections. Med Phys 26:1568-1578.

Jeong EK, Kim SE, Kholmovski EG, Parker DL (2006) High-resolution DTI of a localized volume using 3D single-shot diffusion-weighted STimulated echo-planar imaging (3D ss-DWSTEPI). Magn Reson Med 56:1173-1181.

Kharbanda HS, Alsop DC, Anderson AW, Filardo G, Hackney DB (2006) Effects of cord motion on diffusion imaging of the spinal cord. Magn Reson Med 56:334-339.

Kim JH, Budde MD, Liang HF, Klein RS, Russell JH, Cross AH, Song SK (2006) Detecting axon damage in spinal cord from a mouse model of multiple sclerosis. Neurobiol Dis 21:626-632.

Kim JH, Loy DN, Liang HF, Trinkaus K, Schmidt RE, Song SK (2007) Noninvasive diffusion tensor imaging of evolving white matter pathology in a mouse model of acute spinal cord injury. Magn Reson Med 58:253-260.

Kokolakis G, Panagis L, Stathopoulos E, Giannikaki E, Tosca A, KrügerKrasagakis S (2008) From the protein to the graph: how to quantify immunohistochemistry staining of the skin using digital imaging. J Immunol Methods 331:140-146.

Kornelsen J, Stroman PW (2004) fMRI of the lumbar spinal cord during a lower limb motor task. Magn Reson Med 52:411-414.

Lovas G, Szilágyi N, Majtényi K, Palkovits M, Komoly S (2000) Axonal changes in chronic demyelinated cervical spinal cord plaques. Brain 123:308-317.

Loy DN, Magnuson DS, Zhang YP, Onifer SM, Mills MD, Cao QL, Darnall JB, Fajardo LC, Burke DA, Whittemore SR (2002) Functional redundancy of ventral spinal locomotor pathways. J Neurosci 22:315-323.

Loy DN, Kim JH, Xie M, Schmidt RE, Trinkaus K, Song SK (2007) Diffusion tensor imaging predicts hyperacute spinal cord injury severity. J Neurotrauma 24:979-990.

Mac Donald CL, Dikranian K, Bayly P, Holtzman D, Brody D (2007) Diffusion tensor imaging reliably detects experimental traumatic axonal injury and indicates approximate time of injury. J Neurosci 27:11869-11876.

Markovic-Plese S, McFarland HF (2001) Immunopathogenesis of the multiple sclerosis lesion. Curr Neurol Neurosci Rep 1:257-262.

Matos LL, Stabenow E, Tavares MR, Ferraz AR, Capelozzi VL, Pinhal MA (2006) Immunohistochemistry quantification by a digital computerassisted method compared to semiquantitative analysis. Clinics 61:417-424.

Meyer CR, Moffat BA, Kuszpit KK, Bland PL, Mckeever PE, Johnson TD, Chenevert TL, Rehemtulla A, Ross BD (2006) A methodology for registration of a histological slide and in vivo MRI volume based on optimizing mutual information. Mol Imaging 5:16-23.

Miller DH, Thompson AJ, Filippi M (2003) Magnetic resonance studies of abnormalities in the normal appearing white matter and grey matter in multiple sclerosis. J Neurol 250:1407-1419.

Mispelter J, Lupu M, Briguet A (2006) NMR probeheads for biophysical and biomedical experiments: theoretical principles and practical guidelines. London: Imperial College.

Nair G, Tanahashi Y, Low HP, Billings-Gagliardi S, Schwartz WJ, Duong TQ (2005) Myelination and long diffusion times alter diffusion-tensorimaging contrast in myelin-deficient shiverer mice. Neuroimage 28:165-174.

Naismith RT, Xu J, Tutlam NT, Snyder A, Benzinger T, Shimony J, Shepherd J, Trinkaus K, Cross AH, Song SK (2008) Disability in optic neuritis correlates with diffusion tensor-derived directional diffusivities. Neurology. Advance online publication. Retrieved December 18, 2008. doi: 0:01.wnl.0000335766.22758.cdv1.

Nijeholt GJ, van Walderveen MA, Castelijns JA, van Waesberghe JH, Polman C, Scheltens P, Rosier PF, Jongen PJ, Barkhof F (1998) Brain and spinal cord abnormalities in multiple sclerosis. Correlation between MRI parameters, clinical subtypes and symptoms. Brain 121:687-697.

Papadopoulos D, Pham-Dinh D, Reynolds R (2006) Axon loss is responsible for chronic neurological deficit following inflammatory demyelination in the rat. Exp Neurol 197:373-385.

Petzold A, Eikelenboom MJ, Keir G, Grant D, Lazeron RH, Polman CH, Uitdehaag BM, Thompson EJ, Giovannoni G (2005) Axonal damage accumulates in the progressive phase of multiple sclerosis: three year follow up study. J Neurol Neurosurg Psychiatry 76:206-211.

Qiu D, Tan LH, Zhou K, Khong PL (2008) Diffusion tensor imaging of normal white matter maturation from late childhood to young adulthood: voxel-wise evaluation of mean diffusivity, fractional anisotropy, radial and axial diffusivities, and correlation with reading development. Neuroimage 41:223-232.

Sathornsumetee S, McGavern DB, Ure DR, Rodriguez M (2000) Quantitative ultrastructural analysis of a single spinal cord demyelinated lesion predicts total lesion load, axonal loss, and neurological dysfunction in a murine model of multiple sclerosis. Am J Pathol 157:1365-1376.

Schmierer K, Wheeler-Kingshott CA, Boulby PA, Scaravilli F, Altmann DR, Barker GJ, Tofts PS, Miller DH (2007) Diffusion tensor imaging of post mortem multiple sclerosis brain. Neuroimage 35:467-477.

Song SK, Sun SW, Ramsbottom MJ, Chang C, Russell J, Cross AH (2002) Dysmyelination revealed through MRI as increased radial (but unchanged axial) diffusion of water. Neuroimage 17:1429-1436.

Song SK, Sun SW, Ju WK, Lin SJ, Cross AH, Neufeld AH (2003) Diffusion tensor imaging detects and differentiates axon and myelin degen- 
eration in mouse optic nerve after retinal ischemia. Neuroimage 20:1714-1722.

Song SK, Yoshino J, Le TQ, Lin SJ, Sun SW, Cross AH, Armstrong RC (2005) Demyelination increases radial diffusivity in corpus callosum of mouse brain. Neuroimage 26:132-140.

Sorzano CO, Thévenaz P, Unser M (2005) Elastic registration of biological images using vector-spline regularization. IEEE Trans Biomed Eng 52:652-663.

Stejskal EO, Tanner JE (1965) Spin diffusion measurements: spin echoes in the presence of a time-dependent field gradient. J Chem Phys 42:288-292.

Sun SW, Liang HF, Trinkaus K, Cross AH, Armstrong RC, Song SK (2006) Noninvasive detection of cuprizone induced axonal damage and demyelination in the mouse corpus callosum. Magn Reson Med 55:302-308.
Sun SW, Liang HF, Schmidt RE, Cross AH, Song SK (2007) Selective vulnerability of cerebral white matter in a murine model of multiple sclerosis detected using diffusion tensor imaging. Neurobiol Dis 28:30-38.

Sun SW, Liang HF, Cross AH, Song SK (2008) Evolving Wallerian degeneration after transient retinal ischemia in mice characterized by diffusion tensor imaging. Neuroimage 40:1-10.

Vrenken H, Pouwels PJ, Geurts JJ, Knol DL, Polman CH, Barkhof F, Castelijns JA (2006) Altered diffusion tensor in multiple sclerosis normalappearing brain tissue: cortical diffusion changes seem related to clinical deterioration. J Magn Reson Imaging 23:628-636.

Wujek JR, Bjartmar C, Richer E, Ransohoff RM, Yu M, Tuohy VK, Trapp BD (2002) Axon loss in the spinal cord determines permanent neurological disability in an animal model of multiple sclerosis. J Neuropathol Exp Neurol 61:23-32. 